\title{
A novel method to test for significant trends in extreme values in serially dependent time series
}

\author{
C. Franzke ${ }^{1}$ \\ Received 14 January 2013; revised 26 February 2013; accepted 26 February 2013; published 11 April 2013.
}

[1] We propose a novel method to investigate the statistical significance of trends of extreme values in serially correlated time series based on quantile regression and surrogate data. This method has the advantage over traditional extreme value methods that it takes into account all data points from the time series. We test this method on a temperature time series from the Antarctic Peninsula (Faraday/Vernadsky station), which is highly non-Gaussian and serially correlated. We find evidence for a significant upward nonlinear trend in the extreme cold temperatures (95th percentile) and that most of the observed warming at Faraday/Vernadsky is due to a reduction in cold extremes. Quantile regression can also be used for multivariate regression with external factors. This multivariate regression analysis suggests that $\mathrm{CO}_{2}$ emissions play a large role in the observed trend at Faraday/Vernadsky while also the ozone hole and solar fluctuations play some role. Citation: Franzke, C. (2013), A novel method to test for significant trends in extreme values in serially dependent time series, Geophys. Res. Lett., 40, 1391-1395, doi:10.1002/grl.50301.

\section{Introduction}

[2] Global climate change affects surface climate in many different and nonlinear ways. Many studies show that first order global warming mainly leads to an increase to mean temperature and, to a lesser extent, to changes of the shape of the distribution around this changing mean. However, changes especially in the shape and extremes can have serious effects on society and ecosystems.

[3] Previous studies have mainly concentrated on trends in the mean and, to a lesser extent, on extremes. The latter is usually done using extreme value theory by fitting a generalized extreme value (GEV) distribution with time dependent parameters to annual maxima or by using a threshold exceedance approach [Coles, 2001]. These two approaches need to be adjusted when using serially correlated data [Coles, 2001], and most environmental time series are serially correlated. Furthermore, it has also to be demonstrated that the observed data indeed follow an extreme value distribution. Here, we propose to use quantile regression for trend identification in a given percentile of a serially correlated time series, which does not make any assumptions about the distribution of the data.

\footnotetext{
${ }^{1}$ British Antarctic Survey, Natural Environment Research Council, Cambridge, UK.

Corresponding author: C. Franzke, British Antarctic Survey, Natural Environment Research Council, High Cross, Madingley Road, Cambridge, UK. (chan1@bas.ac.uk)

(C)2013. American Geophysical Union. All Rights Reserved. 0094-8276/13/10.1002/grl.50301
}

[4] The identification of trends in environmental data is impeded because even very simple stationary stochastic processes can exhibit apparent trends over rather long periods of time; so-called stochastic trends [Fatichi et al., 2009; Barbosa, 2011; Franzke, 2010, 2012]. In climate research, one is mainly interested in the identification of trends driven by external forcings like greenhouse gas emissions or solar forcing. These trends are called deterministic trends. In order to distinguish between those two kinds of trends, one has to estimate how often a statistical model for the intrinsic climate fluctuations is able to produce trends, which are larger than the observed trend. The autocorrelation function is one critical component for the duration and amplitude of stochastic trends. In order to test for the presence of deterministic trends, we require statistical models, which capture very well the observed autocorrelation function and amplitude distribution. Most trends in climate are being tested against an autoregressive process null hypothesis, which is Gaussian distributed and thus not suitable for testing for trends in extreme values.

[5] Here, we will show evidence from a long temperature record from the Antarctic station Faraday/Vernadsky [Turner et al., 2004, 2005] that global warming can affect surface temperatures by mainly reducing cold temperature extremes while not simultaneously increasing warm temperatures at the same rate. We do this by quantile regression of the fifth, 50th and 95th percentiles. We propose to use a nonparametric surrogate data method to assess the statistical significance of the changes in the extremes whose surrogate time series simultaneously conserve both the autocorrelation function and distribution of the observed time series. We demonstrate that our novel method finds significant trends in cold extremes, which explains a large part of the observed warming at this station. We also show that quantile regression can be used to identify likely external drivers of the observed trends.

\section{Data and Methods}

[6] To study the changes in extremes, which we define in this study as either the fifth or 95th percentile, we use the well established method of quantile regression [Koenker and Hallock, 2001; Yu et al., 2003; Hannachi, 2006; Barbosa et al., 2011; Donner et al., 2012]. Quantile regression is a regression method, which approximates quantiles of the response variable. Quantile regression is a problem in linear programming, and the simplex algorithm can be used to solve it [Koenker and Hallock, 2001]. For example, linear quantile regression minimizes the functional

$$
\sum_{i=1}^{n} \rho_{p}\left(y_{i}-\mathbf{x}^{T} \beta\right)
$$


a)

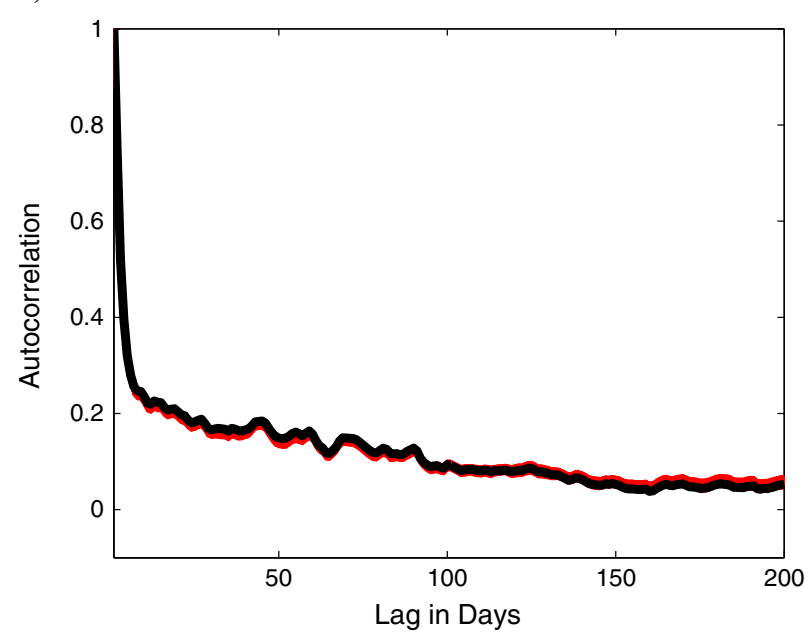

b)

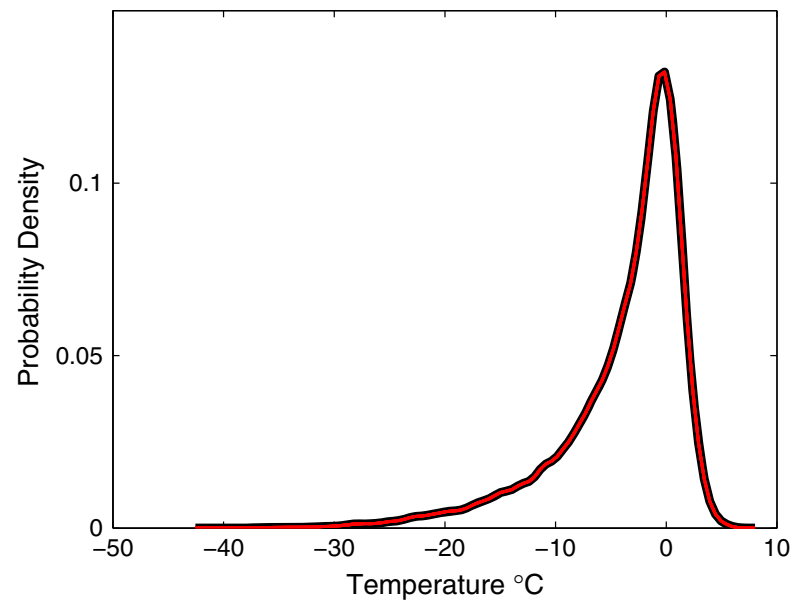

Figure 1. (a) Autocorrelation function and (b) probability density function of the Faraday/Vernadsky station temperature (black line) and of 1000 surrogates (red lines).

where $\rho_{p}(u)=p u I_{[0, \infty)}(u)-(1-p) u I_{(-\infty, 0)}(u)$ is the so-called check function, $I_{A}(u)=1$ if $u \in A$ and otherwise 0 is the indicator function, $n$ is the length of the time series $\mathrm{y}, \mathbf{x}=(1, x)^{T}$, $\mathbf{x}$ is a vector of the time points, but can also contain external factors, $\beta$ denotes the parameter vector to be estimated and $p$ the quantile (see Yu et al. [2003] for more details). Quantile regression can also be used to fit higher order polynomial functions [Koenker and Hallock, 2001].

[7] To test the statistical significance of the quantile trends, we use the surrogate data generating method proposed by Schreiber and Schmitz [1996]. This method generates surrogate time series with the same autocorrelation function and the same probability density function (PDF). This algorithm proceeds as follows: (i) sorted lists of the time series values and the squared Fourier amplitudes are stored, (ii) the time series is randomly shuffled without replacement, (iii) the shuffled time series gets Fourier transformed and then the squared amplitudes are replaced with the ones from the original time series, (iv) after transforming, the shuffled time series back into physical space, it is rank ordered according to the original time series. This rank ordering will affect the Fourier spectrum, so an iteration of steps (iii) and (iv) is required. This iteration continues until the discrepancy in both the Fourier amplitudes and rank ordering becomes sufficiently small.

[8] We demonstrate our approach on daily temperature data from the Faraday/Vernadsky station on the Antarctic Peninsula for the period February 1947 through January 2011 from the Reference Antarctic Data for Environmental Research (READER) data set, which is quality controlled [Turner et al., 2004, 2005]. This temperature time series is highly non-Gaussian (Figure 1b), has a non-trivial decay of its autocorrelation function (Figure 1a) and its cold extremes follow an extreme value distribution, a generalized Pareto distribution [Franzke, 2013].

[9] How well the surrogate time series fit the Faraday/ Vernadsky time series is displayed in Figure 1. For this figure, we generated 1000 surrogate time series and, as can be seen, the Faraday/Vernadsky time series is in the center of the surrogate spread. This suggests that the surrogate time series capture very well the characteristics of the Faraday/Vernadsky time series, especially the highly non-Gaussian PDF. Also, the two time scale decay of the autocorrelation function is very well captured. First, the fast initial decay and then the slower decay starting after about a week. This slower decay is the imprint of the long-range dependence property of the temperature time series reported in Franzke [2010]. The distribution of the fifth, 50th and 95th percentiles regression trend ranges of these quantile regression fits of the surrogate time series is as expected centered around zero (Figure 2). The trend range is defined as the difference between the end and start points of the time series. This confirms that the phase randomization of the original time series has effectively destroyed any trends.
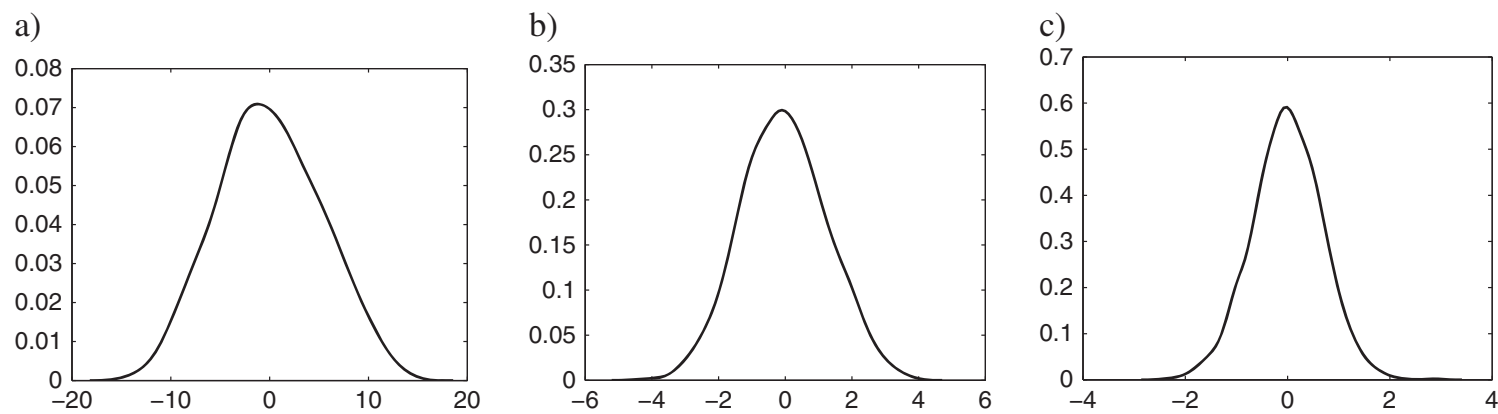

Figure 2. Probability density functions of the trends of (a) fifth percentile, (b) 95th percentile and (c) 50th percentile derived from the 1000 surrogate time series based on the Faraday/Vernadsky station temperature time series. 
a)

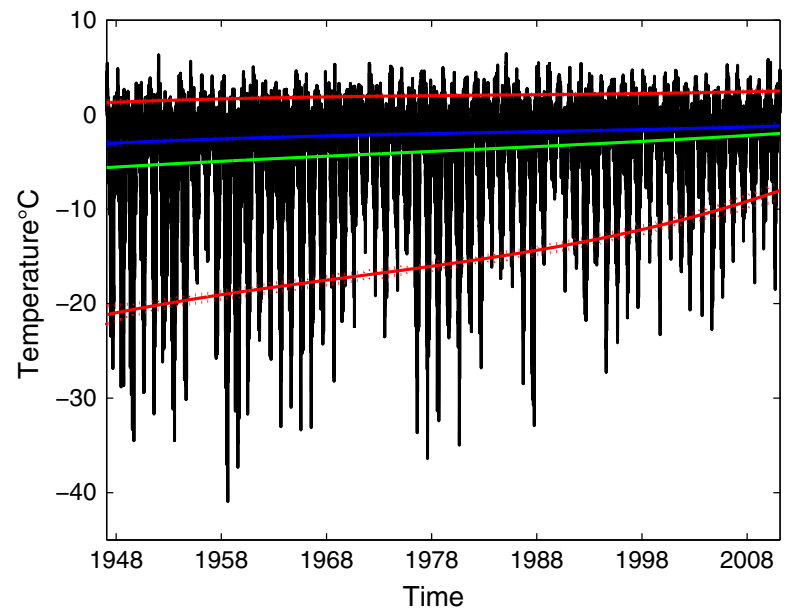

b)

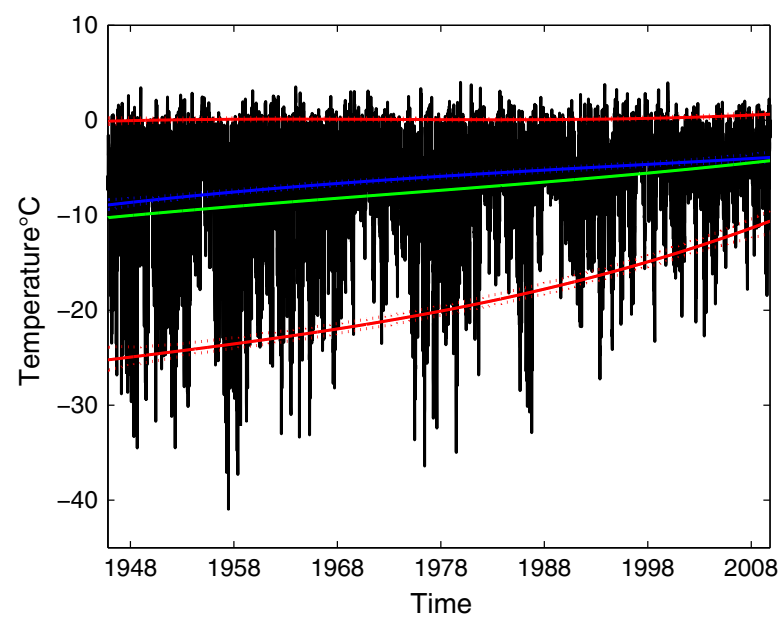

c)

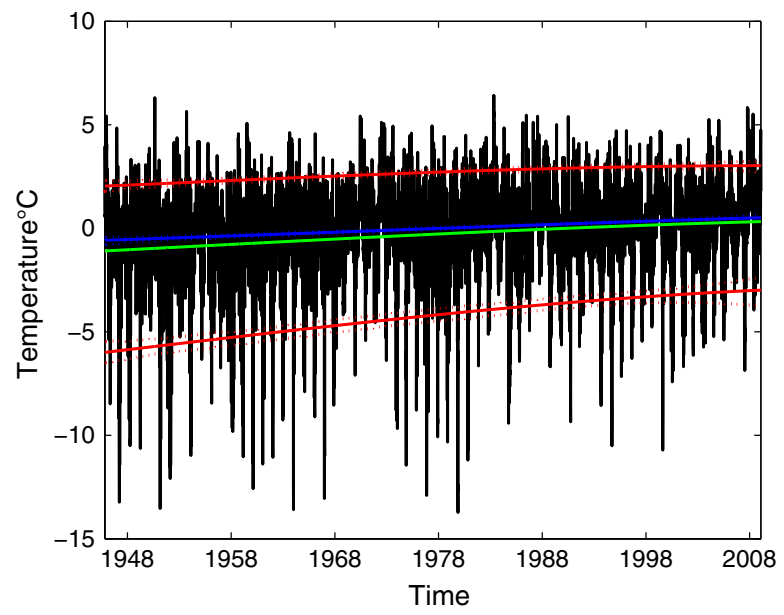

Figure 3. Faraday/Vernadsky station temperature time series (black line), cubic quantile regression of the 50th percentile (blue line), fifth and 95th percentiles (red lines) and cubic linear least-squares regression line (green line). (a) Annual, (b) Winter and (c) Summer. Dotted lines correspond to the $5 \%$ and $95 \%$ confidence levels of the quantile regression derived from a bootstrap approach.
Table 1. Quantile Regression Trend Range ${ }^{\mathrm{a}}$

\begin{tabular}{lccc}
\hline & 5th Percentile & 50th Percentile & 95th Percentile \\
\hline Annual & $\mathbf{2 . 0 2}(\mathbf{1 . 3 6})$ & $0.28(0.33)$ & $\mathbf{0 . 1 9}(\mathbf{0 . 1 7})$ \\
Winter & $\mathbf{2 . 2 5}(\mathbf{1 . 3 4})$ & $\mathbf{0 . 7 7 ( 0 . 7 6 )}$ & $0.11(0.35)$ \\
Summer & $\mathbf{0 . 4 6}(\mathbf{0 . 4 2})$ & $\mathbf{0 . 1 7 ( 0 . 1 5 )}$ & $0.15(0.19)$ \\
\hline
\end{tabular}

${ }^{\mathrm{a}}$ Quantile regression trend range in ${ }^{\circ} \mathrm{C} /$ decade over the data period (1947-2011); in parentheses, the corresponding 95th percentile quantile regression trend range of a 1000 member surrogate time series ensemble. Bold values are significant at the $95 \%$ level.

Table 2. Best Model Fit According to Akaike Information Criterion $^{\mathrm{a}}$

\begin{tabular}{lccc}
\hline & 5th Percentile & 50th Percentile & 95th Percentile \\
\hline Annual & $\mathbf{C O}_{2}+\mathbf{F 1 0 . 7}$ & OMD+F10.7 & $\mathbf{C O}_{2}+$ OMD \\
Winter & $\mathbf{C O}_{2}+\mathbf{F 1 0 . 7}$ & $\mathbf{C O}+\mathbf{O M D}$ & $\mathrm{CO}_{2}+$ OMD + F10.7 \\
Summer & $\mathbf{C O}_{2}+\mathbf{F 1 0 . 7}$ & $\mathbf{C O}_{2}+$ OMD + F10.7 & $\mathrm{CO}_{2}+$ OMD \\
\hline
\end{tabular}

${ }^{a}$ Bold values indicate cases where there is a significant trend present in the Faraday/Vernadsky time series.

Thus, this surrogate method can be used to test for the presence of trends not only in the mean but also in extreme percentiles.

[10] As external factors, we use the $\mathrm{CO}_{2}$ data from the Mauna Loa station (available at http:/www.esrl.noaa.gov/ gmd/ccgg/trends/), the Ozone mass deficit (OMD, which is derived from Total Ozone Mapping Spectrometer (TOMS) measurements, with seasonal, latitudinal and trend corrections to fit the available Dobson measurements [Roscoe and Haigh, 2007]), the $\mathrm{F} 10.7 \mathrm{~cm}$ solar radio flux (available at http://www.drao-ofr.hia-iha.nrc-cnrc.gc.ca /icarus/www/sol\%5Fhome.html) and Southern Hemispheric Stratospheric Aerosol Optical Depth (SAOD; available at http://data.giss.nasa.gov/modelforce/strataer/). We also used solar irradiance, but because it is highly correlated with F10.7 (correlation value is about 0.8 ) and does not change the results qualitatively, we do not report those results. Because the OMD data started in 1979, we use these external factors only for the period 1979-2009. For this period, we have an overlap of all used data. The external factors are effectively independent from each other, so we do not have to take account of any dependence structure in the regression analysis.

\section{Results}

[11] Recent studies provide evidence that observed temperature trends are well described by cubic polynomials [Franzke, 2012]. Thus, we use cubic quantile regression in this study. The cubic quantile regression of the Faraday/ Vernadsky temperature time series shows clear upward trends of the quantile regression lines for the fifth (cold extremes) and 50th percentiles (median) and only a slight upward trend for the 95th percentile (warm extremes). This is the case for the annual period (Figure 3 ) as well as the summer (November through March) and winter (May through September) seasons (Figure 3). Also the bootstrapped confidence levels [Koenker and Hallock, 2001] of the quantile regression are very tight providing confidence in the robustness of the fits. Linear quantile regression trends show similar upward trends (not shown). 
[12] In order to test if the observed upward trends in the percentiles are unlikely to have arisen from intrinsic climate fluctuations, we use a bootstrap approach using surrogate data. We generate 1000 surrogate time series realizations to represent intrinsic climate fluctuations and ask how many times the observed trends are outside of the distribution of trends in the surrogate data [Franzke, 2012]. We claim to have found evidence for a significant warming trend when the observed trend is outside of the 95th percentile of the distribution of the trends of the surrogate data.

[13] This test shows that the upward trend of the 95th percentile is unlikely to stem from intrinsic climate fluctuations for the annual period as well as the summer and winter seasons (Table 1). The 50th percentile upward trend is significant in the summer and winter seasons while the 95th percentile trend is significant for the annual period (Table 1). Thus, the reduction in extreme cold events (fifth percentile) is very robust while the increases in the 50th and 95th percentiles are more seasonal dependent.

[14] Our results reveal that the fifth percentile of the annual temperature has increased by about $14^{\circ} \mathrm{C}$ over the data period, which is about $2^{\circ} \mathrm{C}$ per decade. This increase occurred mainly in the winter season and to a lesser extent during summer. This increase is largely responsible for the observed warming at Faraday-Vernadsky [Turner et al., 2005; Franzke, 2010]. The median and the 95th percentiles have increased by much lesser amounts, by $1.8^{\circ} \mathrm{C}$ and $1.23^{\circ} \mathrm{C}$ over the data period, respectively.

[15] Next, we investigate whether external factors covary with the increases in the fifth, 50th and 95th percentiles. If external factors covary with the observed increases, then this would be a first step in attributing these external factors to have caused the increase. For this we computed multivariate quantile regression fits for all possible combinations of the above external factors and used the Akaike Information Criterion (AIC; von Storch and Zwiers [1999]) to determine the most parsimonious regression model explaining the observed changes in the Faraday/Vernadsky temperature time series. This linear multivariate quantile regression analysis reveals that the best models according to the AIC consist of external factor combinations, which include $\mathrm{CO}_{2}$ (Table 2). All significant percentile trends are related to $\mathrm{CO}_{2}$. Also, external factor combinations including OMD and F10.7 provide good model fits according to AIC and thus these variables also played roles in the increases but not as consistently as $\mathrm{CO}_{2}$. Our analysis shows no evidence for SAOD playing a role in the increase of the amplitude of the fifth percentile. The difference in AIC between the best fit models and the second best is about 2 orders of magnitude. Moreover, the difference in AIC between the best models and the model without any external factors is about 3 orders of magnitude. This provides strong evidence that the recent observed temperature trends at Faraday/Vernadsky are due to external factors.

[16] The most parsimonious external factor combination explaining the quantile trend in the extreme cold temperatures (fifth percentile) is by the combination of $\mathrm{CO}_{2}$ and F10.7 (Table 2). While for the significant median and the 95th percentile trends, the models always include the combination of $\mathrm{CO}_{2}$ and OMD. In summer, the median trend is best explained by the external factor combination of $\mathrm{CO}_{2}$, OMD and F10.7.
[17] The observed increase of the cold extremes (fifth percentile) is likely related to the observed changes and the trend in the Southern Annular Mode (SAM; Marshall [2003]; Franzke [2009]) and/or the observed change in the non-annular atmospheric circulation [Turner et al., 2009]. Climate model simulations have attributed these changes to stratospheric ozone loss and greenhouse gas emissions. The Antarctic Peninsula is characterized by a North-South oriented mountain range. The wind flows mainly parallel to this range. The observed large-scale circulation changes have likely led to a preference of poleward wind flow advection of relative mild air from the Southern ocean and consequently to a reduction of equatorward advection of cold air from the Antarctic interior.

\section{Summary}

[18] We propose a novel method to test for trends in extremes in serially correlated time series by using quantile regression and a surrogate data approach, which produces surrogate time series with the same autocorrelation structure and amplitude distribution. The major advantage of this method is that it takes into account all data and not only values above a predefined threshold. While in this study, we used both linear and cubic quantile regression; it can also be used in a fully non-parametric way [Donner et al., 2012].

[19] Our results provide evidence that at Faraday/ Vernadsky, the observed warming is mainly associated with a reduction in cold extremes. which is consistent with recent findings [Hughes et al., 2007; Turner et al., 2012; Franzke, 2013]. The warm temperatures increase to a much lesser extent, and this change is only significant when using data throughout the year and not during the summer or winter season.

[20] While a regression analysis does not provide strict proof of causal relationships, our results nonetheless suggests that the increase in $\mathrm{CO}_{2}$ plays a major role in the reduction of cold extremes, while the ozone hole and solar fluctuations (F10.7) also play some role. Our statistical attribution results can be used as a baseline for attribution studies with numerical climate models.

[21] Acknowledgments. I thank H. Roscoe for providing me with the OMD data and R. Donner for very helpful comments on an earlier version of the manuscript. This study is part of the British Antarctic Survey Polar Science for Planet Earth Programme. It was funded by the Natural Environment Research Council.

\section{References}

Barbosa, S. M., M. G. Scotto, and A. M. Alonso (2011), Summarising changes in air temperature over Central Europe by quantile regression and clustering, Nat. Hazards Earth Syst. Sci., 11, 3227-3233, doi: 10.5194/nhess-11-3227-2011.

Barbosa, S. M. (2011), Testing for Deterministic Trends in Global Sea Surface Temperature, J. Climate, 24, 2516-2522, doi: 10.1175/2010JCLI3877.1.

Coles, S. (2001), An Introduction to Statistical Modelling of Extreme Values, 208p, Springer, London.

Donner, R. V., R. Ehrcke, S. M. Barbosa, J. Wagner, J. F. Donges, and J. Kurths (2012), Spatial patterns of linear and nonparametric long-term trends in Baltic sea-level variability, Nonlin. Processes Geophys., 19 , 95-111, doi:10.5194/npg-19-95-2012.

Fatichi, S., S. M. Barbosa, E. Caporali, and M. E. Silva (2009), Deterministic versus stochastic trends: Detection and challenges, J. Geophys. Res., 114, D18121, doi:10.1029/2009JD011960.

Franzke, C. (2009), Multi-scale analysis of teleconnection indices: Climate noise and nonlinear trends, Nonlin. Pro. Geophys., 16, 65-76, doi: 10.5194/npg-16-65-2009. 
Franzke, C. (2010), Long-range dependence and climate noise characteristics of Antarctic temperature data, J. Climate, 23, 6074-6081, doi: 10.1175/2010JCLI3654.1.

Franzke, C. (2012), Nonlinear trends, long-range dependence and climate noise properties of surface air temperature, J. Climate, 25, 4172-4183, doi:10.1175/JCLI-D-11-00293.1.

Franzke, C. (2013), Significant reduction of cold temperature extremes in the Antarctic Peninsula at Faraday/Vernadsky Station, Int. J. Clim, doi: $10.1002 /$ joc. 3490 .

Hannachi, A. (2006), Quantifying changes and their uncertainty in probability distributions of climate variables using robust statistics, Clim. Dyn., 27, 301-317, doi:10.1007/s00382-006-0132-X.

Koenker, R., and K. F. Hallock (2001), Quantile regression, J. Economic Prespectives, 15, 143-156.

Hughes, G. L., S. S. Rao, and T. S. Rao (2007), Statistical analysis and time-series models for minimum/maximum temperatures in the Antarctic Peninsula, Proc. R. Soc. A., 463, 241-259, doi:10.1098/rspa.2006.1766.

Marshall, G. J. (2003), Trends in the Southern annular mode from observations and reanalyses, J. Climate, 16, 4134-4143, http://dx.doi. org/10.1175/1520-0442(2003)016<4134:TITSAM>2.0.CO;2.

Roscoe, H. K., and J. D. Haigh (2007), Influences of ozone depletion, the solar cycle and the QBO on the Southern Annular Mode, Q. J. R. Meteorol. Soc., 133, 1855-1864, doi:10.1002/qj.153.

Schreiber, T., and A. Schmitz (1996), Improved surrogate data for nonlinearity tests, Phys. Rev. Lett., 77, 635-638. von Storch, H., and F. W. Zwiers (1999), Statistical Analysis in Climate Research, 484pp, Cambridge University Press, New York.

Turner, J., S. R. Colwell, G. J. Marshall, T. A. Lachlan-Cope, A. M. Carleton, P. D. Jones, V. Langun, P. A. Reid, and S. Iagovkina (2004), The SCAR READER Project: Toward a high-quality database of mean Antarctic meteorological observations, J. Climate, 17, 2890-2898, http://dx.doi.org/10.1175/1520-0442(2004) 017<2890:TSRPTA>2.0.CO;2.

Turner, J., S. R. Colwell, G. J. Marshall, T. A. Lachlan-Cope, A. M. Carleton, P. D. Jones, V. Langun, P. A. Reid, and S. Iagovkina (2005), Antarctic climate change during the last 50 years, Int. J. Climatol., 25, 279-294, doi:10.1002/joc.1130.

Turner, J., J. C. Comiso, G. J. Marshall, T. A. Lachlan-Cope, T. Bracegirdle, T. Maksym, M. P. Meredith, Z. Wang, and A. Orr (2009), Non-annular atmospheric circulation change induced by stratospheric ozone depletion and its role in the recent increase of Antarctic sea ice extend, Geophys. Res. Lett., 36, L08502, doi:10.1029/2009GL037524.

Turner, J., T. Maksym, T. Phillips, G. J. Marshall, and M. P. Meredith (2012), The impact of changes in sea ice advance on the large winter warming on the western Antarctic Peninsula, Int. J. Clim., doi: 10.1002/joc. 3474

Yu, K., Z. Lu, and J. Stander (2003), Quantile regression: application and current research areas, The Statistician, 52, 331-350, doi: 10.1111/14679884.00363 . 